\title{
Análisis de la prevalencia de homofobia en población joven: repercusión en la calidad de vida
}

\author{
Marcel Elipe-Miravet \\ al226029@uji.es \\ Juan Enrique Nebot-García \\ junebot@uji.es \\ Ana Antelo-García \\ al386592@uji.es \\ Marta García-Barba \\ barbam@uji.es \\ Rafael Ballester-Arnal \\ rballest@uji.es
}

\section{Resumen}

Introducción: Aunque en las últimas décadas se han aprobado leyes a favor de la diversidad sexual, la homofobia y la discriminación hacia el colectivo LGTBI siguen siendo habituales en nuestra sociedad. Sin embargo, en la literatura no se ha analizado todavía con suficiente atención. Nuestro objetivo es comprobar si el bienestar de los jóvenes está relacionado con su orientación sexual. Además, se quiere averiguar si los participantes han sufrido algún tipo de discriminación o violencia física o verbal por su orientación sexual.

Método: 602 personas (221 hombres y 381 mujeres), con media de edad de 21,78 años $(D T=6,52)$ cumplimentaron una encuesta sobre experiencia y prácticas sexuales. El $90 \%$ se consideraban heterosexuales y el $10 \%$ homosexuales.

Resultados: Los homosexuales tienen menor satisfacción con la vida en general, con su cuerpo y con su vida sexual. Entre otros aspectos, los homosexuales presentan bajo deseo sexual comparado con los heterosexuales. Asimismo, los homosexuales sufren mayor discriminación y más violencia.

Conclusiones: La homofobia sigue siendo una lacra de nuestra sociedad. A pesar de los avances realizados, es un hecho que el colectivo LGTBI es discriminado, tanto en el ámbito social como personal, y esto afecta a su calidad de vida y a su bienestar. Es necesario profundizar en las variables implicadas para ajustar los programas de intervención. 
Palabras clave: orientación sexual, satisfacción vital, discriminación, violencia, homofobia.

\begin{abstract}
Introduction: Although in the last decades laws have been passed in favor of sexual diversity, homophobia and discrimination towards LGBTI collective is still common in our society. However, in the literature it has not yet been analyzed in detail. Our aim is to check whether the well-being of young people is related to their sexual orientation. In addition, we want to find out if the participants have suffered any type of discrimination and physical or verbal violence because of their sexual orientation.

Method: 602 people (221 men and 381 women), with a mean of 21.78 years old $(S D=6,52)$, completed a survey on sexual experience and practices. $90 \%$ considered themselves as heterosexual and the other $10 \%$ were homosexual. Results: homosexuals have lower satisfaction with their life, their body and finally with their sex life. Among other aspects, homosexuals have low sexual desire compared with heterosexuals. Likewise, homosexuals suffer more discrimination and more violence.

Conclusions: Homophobia remains a scourge of our society. In spite of the progress made, it is a fact that LGBTI collective is harshly discriminated, both socially and personally, affecting their life quality and their happiness. It is necessary to delve into the involved variables in order to adjust the intervention programs.
\end{abstract}

Keywords: sexual orientation, life satisfaction, discrimination, violence, homophobia.

\title{
Introducción
}

Según el último informe de la International Lesbian, Gay, Bisexual, Trans and Intersex Association (ILGA), España ha dejado de estar entre los 10 países europeos más respetuosos con el colectivo LGTBI (ILGA 2019). En el 2010, año en que se realizó el primer informe, España se situaba prácticamente en la cabeza, ocupando el segundo lugar. Sin embargo, desde entonces, nuestro país no ha dejado de caer en picado y se sitúa actualmente en la decimoprimera posición. El estancamiento en cuanto a la aprobación de nuevas leyes que garanticen la igualdad y el respeto hacia este colectivo parece ser la principal causa de este descenso. No es de extrañar si se tiene en cuenta que en esta última década el Gobierno del país ha sido principalmente de ideología conservadora. Además, la reciente entrada en el Congreso de la extrema derecha hace que las predicciones no sean tan halagüeñas como muchos esperan.

Dejando de un lado la política, diversos estudios muestran cómo el colectivo LGTBI sufre mayores ratios de discriminación (García Barba, Castro Calvo, Salmerón Sánchez y Ballester Arnal 2017), menor apoyo de la sociedad (Garchitorena 2009) y más problemas familiares (Kuyper y Vanwesenbeeck 2011). Estos hallazgos no solo se encuentran en población joven, sino que población adulta también experimenta esta discriminación y estos actos violentos (Nebot García, Giménez García, Ruíz Palomino, Iglesias Campos y Ballester Arnal 2018). Resulta llamativo, por ejemplo, el comportamiento del personal sanitario hacia ellos. Diversos estudios apuntan que 
muchas personas del colectivo LGTBI sufren distintos tipos de discriminaciones, entre ellos la falta de conocimiento sobre salud sexual por parte de los profesionales de la salud, comentarios despectivos sobre su colectivo, excesiva proximidad física o tocamientos innecesarios, así como otros actos discriminatorios, tanto implícitos como explícitos, por parte de médicos, enfermeros e incluso psicólogos (Irwin 2007; Ross y Setchell 2019; Shelton y Delgado-Romero 2011). Ante la discriminación generalizada hacia el colectivo LGTBI, no es de extrañar que haya mayores tasas de suicido con respecto a la población heterosexual por el mero hecho de ser homosexuales. El estudio llevado a cabo por Ream (2019) en población estadounidense muestra cómo el $24 \%$ de las personas de entre 12 y 14 años que se suicidaron eran población LGTBI. Además, el metaanálisis realizado por Yildiz (2018) revela que los jóvenes pertenecientes a grupos sexuales minoritarios presentan mayores tasas de ideación suicida y más intentos de suicidio y tienen mayor riesgo de consumar el acto suicida que los heterosexuales.

Muchas de las personas no heterosexuales también presentan menor satisfacción en varias dimensiones de su vida. Una de ellas es con el propio cuerpo. En comparación con la población heterosexual, el colectivo LGTBI informa peores evaluaciones de su apariencia física, mayor preocupación por su peso, más efectos negativos de su imagen corporal en su calidad de vida y concretamente en la calidad de su vida sexual, menor comodidad al usar traje de baño en público y menor disposición a revelar aspectos de su cuerpo a su pareja durante el acto sexual (Morrison, Morrison y Sager 2004; Peplau et al. 2009).

La vida sexual es otro aspecto que se ve comúnmente afectado. Aunque hay estudios que no encuentran que los homosexuales tengan menor satisfacción sexual (Kuyper y Vanwesenbeeck 2011; McClelland 2011), otros sí encuentran tales diferencias (Gil 2007; Henderson, Lehavot y Simoni 2009). Es el caso de la investigación realizada por Graugaard, Giraldi, Frisch, Falgaard Eplov y Davidsen (2015), quienes observaron que los hombres daneses homosexuales tenían menor satisfacción sexual que los heterosexuales. De la misma forma, tanto hombres como mujeres homosexuales afirmaban que no se satisfacían sus necesidades sexuales, de la misma forma que informaron haber sufrido algún acto de violencia sexual. El hecho de tener una satisfacción sexual más baja puede dar lugar a ciertas disfunciones sexuales. EI metaanálisis llevado a cabo por Grabski y Kasparek (2017) revela que las disfunciones más comunes encontradas en población no heterosexual son la eyaculación retardada, problemas en la erección, dolor en el acto sexual y la no experimentación de placer durante las relaciones sexuales.

Todo lo tratado en los párrafos anteriores apunta hacia una posible menor satisfacción con la vida general. Como muestra de ello encontramos el estudio de McCabe, Hughes, West, Veliz y Boyd (2019), quienes encontraron que la población homosexual estadounidense tenía menor satisfacción vital. Además, este mismo estudio encontró que las mujeres lesbianas y bisexuales tienen una probabilidad mucho más elevada que las mujeres heterosexuales de sufrir trastorno por consumo de alcohol. Pero entre las personas no heterosexuales también hay diferencias. Así, el estudio de Nam, Jun, Fedina, Shah y DeVylder (2019) encuentra que los bisexuales llegan a sufrir incluso mayor estrés, depresión, ansiedad e ideación suicida que los gays y las lesbianas. En muestra española encontramos resultados similares a los estudios anteriores. El estudio de Espada, Morales, Orgilés y Ballester Arnal (2012), llevado a cabo con muestra adolescente, pone de manifiesto cómo la población no heterosexual tiene más riesgo de padecer depresión que la población heterosexual.

Por lo tanto, una vez revisada la bibliografía sobre esta temática, el objetivo del presente trabajo fue comprobar si el bienestar de la población universitaria 
homosexual está relacionado con su identidad sexual. Además, se quiso averiguar si la muestra de este estudio ha sufrido algún tipo de discriminación o violencia, tanto física como verbal, por su propia orientación sexual.

A partir de la revisión que se ha hecho de la literatura sobre esta temática, las hipótesis que se plantean son las siguientes: 1) en nuestra muestra de estudio los jóvenes homosexuales informarán menor satisfacción, tanto con su cuerpo como con su vida sexual y con su vida en general, que los heterosexuales; 2) los participantes que afirmen ser homosexuales habrán experimentado en mayor medida algún tipo de violencia, tanto física como verbal; 3) la población homosexual, en comparación con la heterosexual, sufrirá o habrá sufrido mayores tasas de alguna disfunción sexual, como bajo deseo sexual, problemas de erección y dolor en el coito.

\section{Método}

\section{Participantes}

La muestra estaba formada por un total de 602 participantes, de los cuales 221 eran hombres y 381 fueron mujeres, con una media de edad de 21,78 años (DT=6,52). Del total de la muestra, el $90 \%$ (541) se consideraba heterosexual, mientras que el $10 \%$ (61) restante decía ser homosexual.

\section{Instrumento}

Los participantes cumplimentaron un cuestionario ad hoc elaborado por el grupo de investigación Salusex de la Universitat Jaume I. El cuestionario recogía las siguientes variables: género, edad, orientación sexual, satisfacción personal, satisfacción vital, satisfacción sexual, problemas en el nivel de deseo sexual y discriminación sufrida por la propia orientación sexual. Las variables de satisfacción y discriminación estaban medidas en escala Likert (1-4). Los problemas de bajo deseo sexual, erección y dolor durante el coito se evaluaron mediante escala dicotómica (Sí/No). Por último, las variables de orientación sexual y género eran de distintas opciones de respuesta, mientras que la edad era de respuesta abierta.

\section{Procedimiento}

Los datos fueron recogidos durante los años 2016 y 2017, en concreto en el contexto de campañas de promoción de la salud sexual realizadas en el campus universitario con motivo de la conmemoración del día mundial contra el sida. La aplicación de los cuestionarios (en formato lápiz y papel) fue individual, aunque en lugares públicos. Aun así, los encargados de la aplicación del cuestionario se aseguraron de mantener el rigor exigido en toda investigación, separando debidamente a los participantes con el fin de mantener el anonimato y la confidencialidad de sus respuestas, así como solucionar cualquier duda que pudiera surgir. La participación fue voluntaria.

\section{Análisis estadísticos}

Los datos se analizaron mediante el programa estadístico SPSS (versión 25). Se realizaron análisis descriptivos para estudiar los datos sociodemográficos de los participantes. Posteriormente, se utilizaron contrastes chi cuadrado para ver las diferencias en la satisfacción con diferentes aspectos, en el nivel de deseo sexual y, 
finalmente, en la discriminación y violencia sufrida en función de la orientación sexual de los participantes.

\section{Resultados}

En primer lugar, se analizaron las variables relacionadas con la discriminación y la violencia sufrida. En lo que se refiere a violencia verbal, se ha comprobado que los homosexuales sufren más insultos que los heterosexuales (véase la figura 1), siendo estas diferencias estadísticamente significativas $\left(x^{2}=306,15 ; p<0,001\right)$. Se observa que el $66 \%$ de los homosexuales alguna vez han sufrido insultos o faltas de respeto por su orientación sexual, frente al $5 \%$ de los heterosexuales.

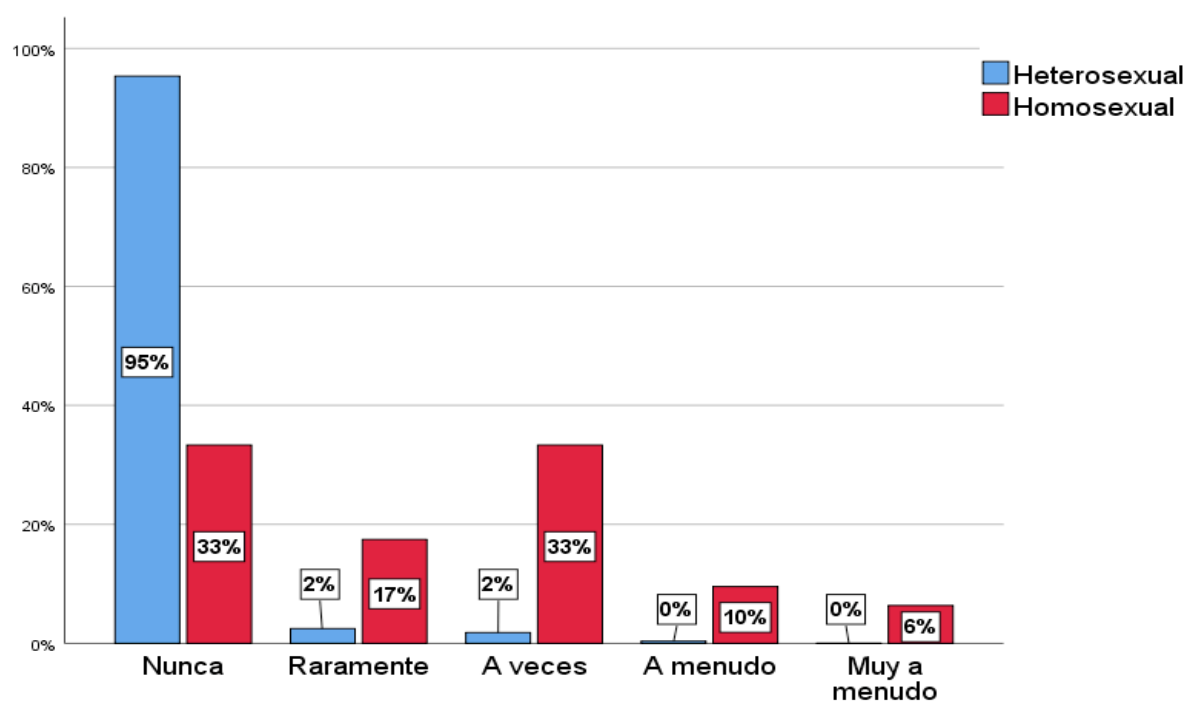

Figura1. Prevalencia de insultos y menosprecios sufridos según orientación sexual.

De igual forma, este colectivo recibe un mayor número de burlas (véase la figura 2) que los heterosexuales $\left(x^{2}=329,80 ; p<0,001\right)$, llegando el porcentaje al $71 \%$, frente solamente al $6 \%$ de los heterosexuales. 


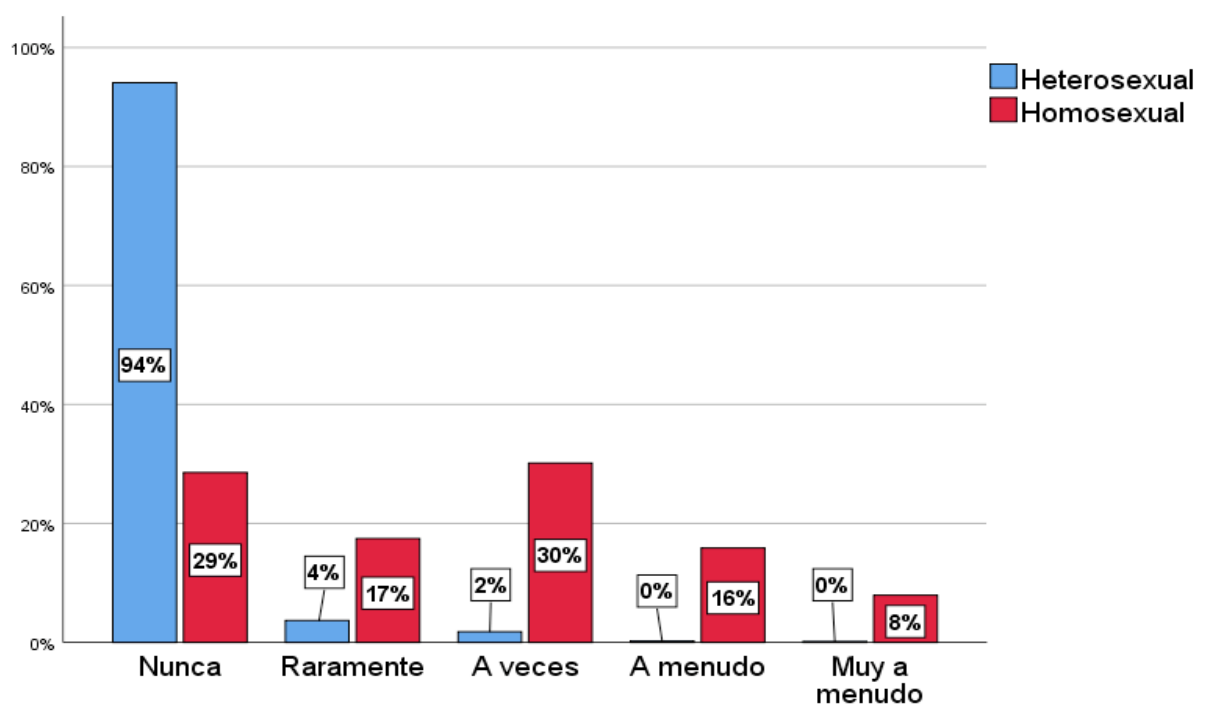

Figura 2. Prevalencia de burlas y mofas sufridas según orientación sexual.

Así mismo, en la figura 3 se observa que el $35 \%$ de los homosexuales alguna vez han sufrido amenazas por su orientación sexual, frente al $2 \%$ de la muestra heterosexual, siendo estas diferencias significativas $\left(x^{2}=157,71 ; p<0,001\right)$.

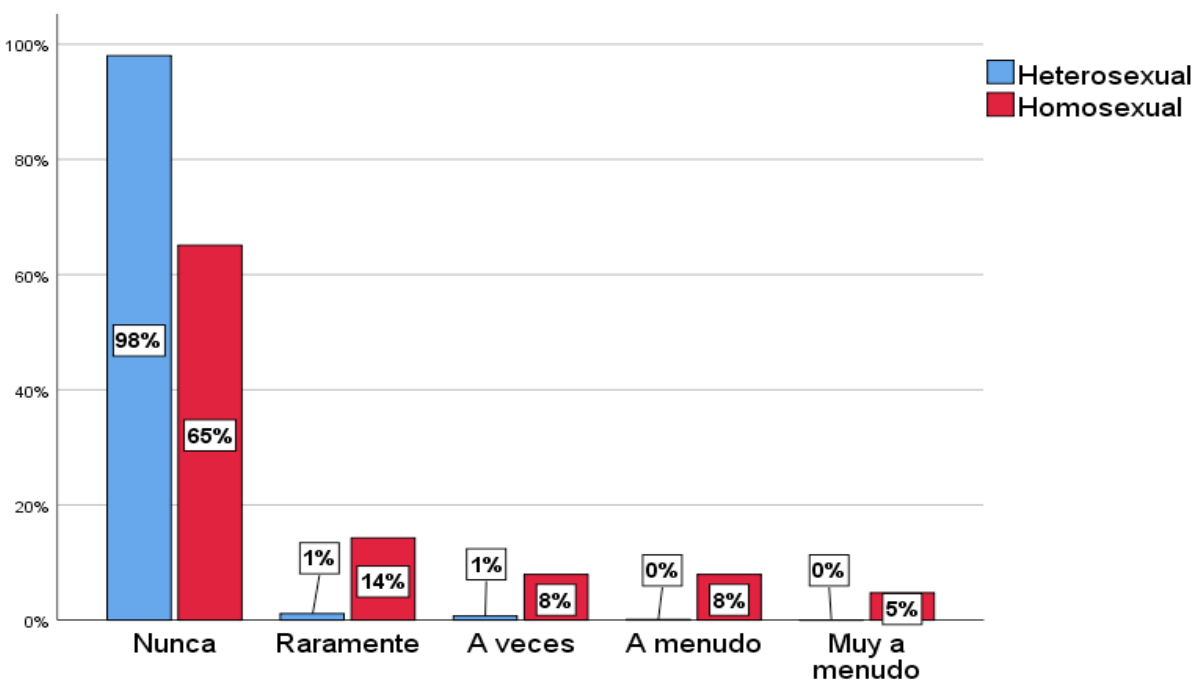

Figura 3. Prevalencia de amenazas sufridas según orientación sexual.

En lo referente a violencia física, el $11 \%$ de mujeres y hombres homosexuales reportan un mayor número de golpes que los heterosexuales $\left(x^{2}=124,39 ; p<0,001\right)$, de la misma forma que el $8 \%$ ha sufrido más palizas indiscriminadas (véase la figura 4) simplemente por su orientación sexual $\left(x^{2}=53,53 ; p<0,001\right)$. 


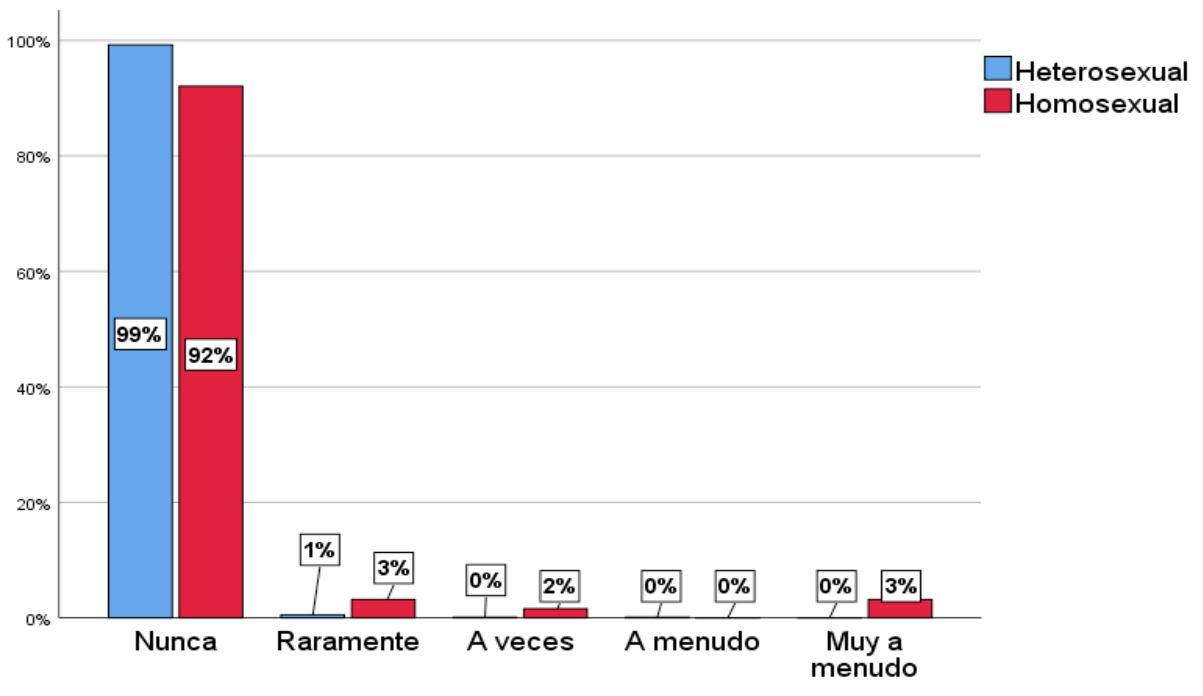

Figura 4. Prevalencia de palizas sufridas según orientación sexual.

En cuanto al análisis de la satisfacción con diferentes aspectos de su vida, encontramos que los jóvenes homosexuales tienen una menor satisfacción con su cuerpo $\left(x^{2}=14,54 ; p=0,002\right)$, con su vida sexual $\left(x^{2}=11,07 ; p=0,011\right)$ y finalmente con su vida en general $\left(x^{2}=8,81 ; p=0,032\right)$. Sin embargo, no se encontraron diferencias significativas entre heterosexuales y homosexuales en cuanto a la satisfacción con sus genitales $\left(X^{2}=0,55 ; p=0,908\right)$, ni con su carácter o forma de ser $\left(X^{2}=10,80 ; p=0,833\right)$.

Por último, encontramos que, aunque con un tamaño del efecto pequeño, existen diferencias significativas en cuanto al deseo sexual de ambos grupos $\left(X^{2}=5,38\right.$; $p=0,020 ; V=0,057$ ), siendo los homosexuales los que experimentan un deseo sexual más bajo. Finalmente, no se encontraron diferencias significativas a la hora de padecer problemas de erección en los hombres $\left(X^{2}=0,298 ; p=0,591\right)$ o de dolor en el coito en las mujeres $\left(X^{2}=0,01 ; p=0,919\right)$.

\section{Discusión y conclusiones}

En el presente artículo se ha podido comprobar cómo, a pesar de contar con una muestra especialmente joven, el colectivo LGTBI sigue siendo cruelmente castigado por la sociedad y sufre un mayor malestar, pese a vivir en el siglo XXI donde se han promulgado leyes mucho más inclusivas y que fomentan el respeto a los derechos de los grupos sexuales minoritarios.

En este sentido, los resultados aquí mostrados apoyarían la primera hipótesis de nuestra investigación, la cual afirmaba que las personas homosexuales tienen una menor satisfacción corporal, sexual y vital que las heterosexuales. Estos resultados no son diferentes a los encontrados en la bibliografía previa, pues en todos los artículos revisados se llegaron a las mismas conclusiones (Graugaard, Giraldi, Frisch, Falgaard Eplov y Davidsen 2015; McCabe, Hughes, West, Veliz y Boyd 2019; Peplau et al. 2009).

Desafortunadamente, los resultados hallados confirman que se cumple la segunda hipótesis de nuestro estudio, pues nuestra muestra homosexual ha experimentado tanto violencia física como verbal, aunque un porcentaje mucho más elevado son los 
que han sufrido insultos, burlas o amenazas. Aunque en este estudio no se han estudiado las consecuencias psicológicas, no sería extraño encontrar mayores niveles de estrés, ansiedad o depresión en este subgrupo poblacional a consecuencia de la discriminación sufrida, tal y como refleja la bibliografía revisada (Nam, Jun, Fedina, Shah y DeVylder 2019).

En cuanto a la tercera y última hipótesis, podemos decir que se ha cumplido parcialmente. Por una parte, los resultados del estudio sí que revelan diferencias significativas a la hora de experimentar deseo sexual, siendo este estadísticamente más bajo en el grupo de los homosexuales. Ahora bien, no se han encontrado diferencias significativas a la hora de padecer problemas de erección en los hombres o dolor en la penetración en las mujeres. Tanto los resultados relacionados con el dolor como los problemas de erección son contradictorios con los encontrados en el estudio de Grabski y Kasparek (2017), quienes sí encontraron diferencias en distintas disfunciones sexuales que aquí no se han hallado.

Creemos que los resultados aquí hallados están relacionados entre sí. Por una parte, el hecho de haber sufrido algún tipo de violencia genera o ha generado malestar en los participantes. Si estos abusos y menosprecios se han producido en ciertas edades del desarrollo o durante periodos prolongados, sería normal encontrarse con una menor satisfacción general, así como una menor satisfacción corporal y sexual, lo que podría traducirse en un menor deseo sexual que en el grupo de heterosexuales.

A pesar de los hallazgos aquí encontrados, esta investigación está ligada a ciertas limitaciones. La principal y más importante es la baja muestra homosexual con la que cuenta este estudio. Esta característica quizás este limitando los resultados aquí encontrados. De la misma forma, sería interesante introducir participantes bisexuales en los datos, para poder contar con una muestra que cubra un poco más el espectro del colectivo LGTBI. Por lo tanto, las líneas futuras de investigación van encaminadas tanto hacia la ampliación de muestra homosexual como a la inclusión del grupo bisexual y de otras minorías sexuales como los transexuales, pues solamente de esta forma estaríamos contando con una representación fidedigna del colectivo LGTBI. También sería relevante poder añadir variables al cuestionario que aborden el estrés, la ansiedad, la depresión, la ideación suicida y el bienestar global. Así, conseguiríamos explorar más ampliamente el abanico de sentimientos y preocupaciones de la población de estudio, con la finalidad de analizar su bienestar o malestar psicológico y, de esta forma, desarrollar programas de intervención ajustados al perfil psicológico y necesidades de estas minorías sexuales.

\section{Referencias bibliográficas}

Espada, José, Alexandra Morales, Mireia Orgilés y Rafael Ballester Arnal. 2012. «Autoconcepto, ansiedad social y sintomatología depresiva en adolescentes españoles según su orientación sexual». Ansiedd y Estrés, 18(1): 31-41.

Garchitorena, Marta. 2009. Informe Jóvenes GLBT. Federación Estatal de Lesbianas, Gays, Transexuales y Bisexuales (FELGTB). Recuperado de: http://www.felgtb.org

García Barba, Marta, Jesús Calvo Calvo, Pedro Salmerón Sánchez y Rafael Ballester Arnal. 2017. "Orientación sexual y homofobia en adolescentes españoles». Àgora de Salut, 4: 163-172.

Gil, Sharon. 2007. "Body image, well-being and sexual satisfaction: A comparison between heterosexual and gay men». Sexual and Relationship Therapy, 22(2): 237-244. 
Grabski, Barstsz y Krzysztof Kasparek. 2017. "Sexual dysfunctions in nonheterosexual men literature review». Psychiatria Polska, 51(1): 85-94.

Graugaard, Chirstian, Annamaria Giraldi, Morten Frisch, Lene Falgaard Eplov y Michael Davidsen. 2015. "Self-reported sexual and psychosocial health among non-heterosexual Danes». Scandinavian Journal of Public Health, 43(3): 309314.

Henderson, Alison, Keren Lehavot y Jane Simoni. 2009. «Ecological models of sexual satisfaction among lesbian/bisexual and heterosexual women». Archives of Sexual Behavior, 38(1): 50-65.

ILGA. 2019. Annual Review of the Human Rights Situation of Lesbian, Gay, Bisexual, Trans and Intersex People in Europe 2019. Brussels: ILGA-Europe.

Irwin, Lyn. 2007. «Homophobia and heterosexism: Implications for nursing and nursing practice». Australian Journal of Advanced Nursing, 25(1): 70-76.

Kuyper, Lisette e Ine Vanwesenbeeck. 2011. «Examining sexual health differences between lesbian, gay, bisexual, and heterosexual adults: The role of sociodemographics, sexual behavior characteristics, and minority stress». Journal of Sex Research, 48(3): 263-274.

McCabe, Sean, Tonda Hughes, Brady West, Philip Veliz y Carol Boyd. 2019. «DSM-5 Alcohol Use Disorder Severity as a Function of Sexual Orientation Discrimination: A National Study». Alcoholism: Clinical and Experimental Research, 43(3): 497-508.

McClelland, Sara. 2011. "Who is the "Self" in self reports of sexual satisfaction? Research and policy implications». Sexuality Research and Social Policy, 8(4): 304-320.

Morrisson, Melani, Todd Morrisson y Cheryl Sager. 2004. «Does body satisfaction differ between gay men and lesbian women and heterosexual men and women? A meta-analytic review». Body Image, 1: 127-138.

Nam, Boyoung, Hyun Jun, Lisa Fedina, Roma Shah y Jordan DeVylder. 2019. «Sexual orientation and mental health among adults in four U.S. cities». Psychiatry Research, 273: 134-140.

Nebot García, Juan Enrique, Cristina Giménez García, Estefania Ruíz Palomino, Paula Iglesias Campos y Rafael Ballester Arnal. 2018. «Malestar emocional asociado a la orientación sexual en personas mayores». International Journal of Developmental and Educational Psychology. Revista INFAD de Psicología, 4(1): 49.

Peplau, Letitia, David Frederick, Curtis Yee, Natalya Maisel, Janet Lever y Negin Ghavami. 2009. "Body Image Satisfaction in Heterosexual, Gay, and Lesbian Adults». Archives of Sexual Behavior, 38(5): 713-725.

Ream, Geoffrey. 2019. "What's Unique About Lesbian, Gay, Bisexual, and Transgender (LGBT) Youth and Young Adult Suicides? Findings from the National Violent Death Reporting System». Journal of Adolescent Health, 64(5): 602-607.

Ross, Megan y Jenny Setchell. 2019. «People who identify as LGBTIQ+ can experience assumptions, discomfort, some discrimination, and a lack of knowledge while attending physiotherapy: a survey». Journal of Physiotherapy, 65(2): 99-105.

Shelton, Kimber y Edward Delgado-Romero. 2011. «Sexual orientation microaggressions: The experience of lesbian, gay, bisexual, and queer clients in psychotherapy». Journal of Counseling Psychology, 58(2): 210-221.

Yıldız, Erman. 2018. «Suicide in sexual minority populations: A systematic review of evidence-based studies». Archives of Psychiatric Nursing, 32(4): 650-659. 